\section{Buchrezension zu: Homo sapiens}

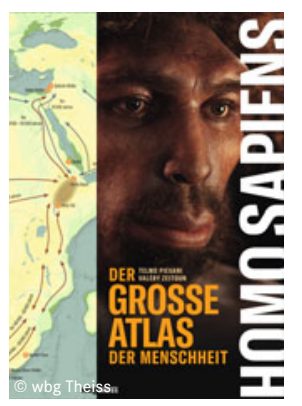

\section{Homo sapiens}

Der große Atlas der

Menschheit

Telmo Pievani und Valéry

\section{Zeitoun}

208 S., wbg Theiss, 2020.

HC mit $S U, 70,-\epsilon$.

ISBN: 9783806242317

DOI: $10.1007 / \mathrm{s} 12268-021-1579-3$

(c) Springer Verlag GmbH 2021

Man schlägt das Buch auf und ist sofort von der tollen Aufmachung gefesselt: viele hervorragende Bilder von archäologischen Funden und Rekonstruktionen früherer Menschen, dazu viele Karten menschlicher Wanderungsbewegungen und Schemazeichnungen, verbunden mit klaren und gut verständlichen Texten über die menschliche Evolution.

Der Text beginnt mit den frühesten archäologischen Funden der Australopthecinen in Afrika und schildert ihre Weiterentwicklung zu Paranthropus und den ersten Formen der Gattung Homo. Bei den drei Auswanderungswellen aus Afrika wurde dann die Route über den Nahen Osten bevorzugt. Die Autoren betonen dabei, dass nur jeweils kleine Gruppen Afrika verlassen haben, also „Gründerpopulationen“ mit einem vergleichsweise kleinen Genpool, was den Effekt hat, dass auch heute noch die genetische Vielfalt der Menschen in Afrika um ein Vielfaches höher ist als außerhalb! Zuletzt trat der anatomisch moderne Mensch an, sich auf der ganzen Welt auszubreiten, und er traf dabei auf die Menschen aus der vorherigen Auswanderungswelle. Das führte zu Phasen, in denen sich mehrere „Menschhei- ten" zur gleichen Zeit am gleichen Ort aufgehalten haben, nämlich auch die Neandertaler und Denisova-Menschen als Nachfahren des Homo antecessor. Aber die verschiedenen Menschenarten hatten sich noch nicht so weit auseinanderentwickelt, dass es nicht zu Vermischungen kommen konnte, wodurch wir Europäer noch genetische Spuren der Neandertaler in uns und die Menschen in Ozeanien Überreste von den Denisova-Menschen tragen.

Der „Atlas der Menschheit“ beschreibt auch unsere kulturelle Entwicklung. Symbolisches Denken drückte sich zunächst in realistischen Jagdszenen aus, Bildhauerei und rituelle Bestattungen entwickelten sich. Landwirtschaft und Viehzucht entstanden zunächst im „fruchtbaren Halbmond" des Mittleren Ostens, in China, Neuguinea und Mittelamerika - verbunden mit Mutationen, die zu Laktosetoleranz bei Erwachsenen führten.

Abschließend diskutieren die Autoren die Entwicklung von Sprache und Schrift. Die Entwicklung der Keilschrift in Anatolien und dem Mittleren Osten lässt sich anhand archäologischer Funde nachverfolgen, aber Linguisten können auch Stammbäume von Sprachen aufstellen. Ein solcher Stammbaum beschreibt die indogermanische Sprachfamilie und ihren Ursprung in Anatolien. Gab es aber auch so etwas wie eine „Weltursprache“? Und wo kommt das Baskische her?

Fazit: ein tolles und vielfältiges Buch, das an vielen Stellen Lust „auf mehr" macht!

Jochen Graw, Unterschleißheim, jochen.graw@tum.de 